This is a post-peer-review, pre-copyedit version of an article published in J ournal of Superconductivity and Novel Magnetism. The final authenticated version is available online at: https://dx. doi.org/10.1007/s10948-016-3474-6 


\title{
Enhanced spin tunneling in a molecular magnet mixed with a superconductor
}

\author{
J. Tejada, R. Zarzuela, A. García-Santiago \\ Grup de Magnetisme, Departament de Física Fonamental, Facultat de Física, \\ Universitat de Barcelona, Martí i Franquès 1, 08028 Barcelona, Spain \\ I. $\operatorname{Imaz}^{1}$, J. Espin ${ }^{1}$, D. Maspoch ${ }^{1,2}$ \\ ${ }^{1}$ Institut Català de Nanotecnologia, ICN2, Esfera Universitat \\ Autónoma Barcelona (UAB), Campus UAB, 08193 Bellaterra, Spain \\ ${ }^{2}$ Institució Catalana de Recerca $i$ Estudis Avançats (ICREA), 08100 Barcelona, Spain \\ E. M. Chudnovsky \\ Physics Department, Lehman College, The City University of New York, \\ 250 Bedford Park Boulevard West, Bronx, NY 10468-1589, USA
}

(Dated: September 25, 2015)

\begin{abstract}
We report characterization and magnetic studies of mixtures of micrometer-size ribbons of $\mathrm{Mn}_{12}$ acetate and micrometer-size particles of $\mathrm{YBaCuO}$ superconductor. Extremely narrow zero-field spintunneling resonance has been observed in the mixtures, pointing to the absence of the inhomogeneous dipolar broadening. It is attributed to the screening of the internal magnetic fields in the magnetic particles by Josephson currents between superconducting grains surrounding the particles.

PACS numbers: 75.50.Xx, 75.45. $+\mathrm{j}, 74.81 . \mathrm{Bd}$
\end{abstract}

\section{INTRODUCTION}

Molecular magnets have been at the forefront of research on quantum spin phenomena at the nanoscale ${ }^{1}$. They provide an ultimate limit of the miniaturization of magnetic memory and are promising candidates for qubits - elements of quantum computers ${ }^{2}$. Quantum effects observed in molecular magnets include quantum tunneling of the magnetic moment ${ }^{3}-6$, topological Berry phase $^{7}$, quantum magnetic deflagration $\frac{89}{}$, and Rabi oscillations 1011 . Most recently it was demonstrated that measurement of the electric current through a magnetic molecule permits readout of quantum states of an individual atomic nucleus $12 \mid 13$

In molecular magnets the inversed population of spin energy levels can be created by simply applying the magnetic field. It was suggested some time ago that this can be used to achieve superradiance and laser effect in molecular magnets 14/15. Besides fundamental interest, such effects would be of significant practical interest as they would provide sources of coherent electromagnetic radiation in the frequency range of a few hundred $\mathrm{GHz}$ which are difficult to obtain by other methods. The subsequent studies ${ }^{16}$, however, revealed that inhomogeneous broadening of spin levels in molecular magnets is a great impediment on the way of achieving coherent radiation. It originates from dipolar and hyperfine interactions, as well as from $D$-strains and $g$-strains 17 . Typically observed widths of the resonances are in the ball park of $1 \mathrm{kOe}$. The zero-field resonance stands out because it is not subject to $D$-strains and $g$-strains and because it also shows the absence of hyperfine broadening 18 , apparently due to the fast transitions between nuclear states on a time-scale of a typical field-sweep experiment. For conventional $\mathrm{Mn}_{12}$ acetate, the typical width of the zero-field resonance is in the ball park of 300 Oe.

In this Letter we report experimental attempt to reduce the width of the zero-field spin-tunneling resonance by mixing micron-size ribbons of $\mathrm{Mn}_{12}$ acetate with micron-size grains of $\mathrm{YBaCuO} . \mathrm{Mn}_{12}$ ribbons with triclinic short-range crystal structure (see below) exhibit more narrow zero-field resonance than conventional $\mathrm{Mn}_{12}$ acetate. Further reduction of the width of the resonance requires elimination of the dipolar broadening. Here we explore the idea of screening the internal dipolar magnetic fields in $\mathrm{Mn}_{12}$ particles by superconducting currents in the $\mathrm{YBaCuO}$ grains surrounding the particles 19 . In accordance with our expectation, we observed a pronounced magnetic relaxation in the vicinity of zero field and the width of the zero-field resonance reduced to values as low as 25 Oe.

Ribbon-shaped $\mathrm{Mn}_{12}$-acetate particles were prepared by re-precipitation 20 of $\mathrm{Mn}_{12}$-acetate crystals of size $4.9 \pm 1.0 \mu \mathrm{m}$ that were synthesized as previously described by Lis 21 . We first dissolved $60-\mathrm{mg} \mathrm{Mn}_{12}$-acetate crystals in $15 \mathrm{~mL}$ of acetonitrile, filtered the solution to avoid any solid trace, and mixed it with $30 \mathrm{~mL}$ of toluene under continuous stirring. After one hour a brown solid precipitate was collected by filtration. Its field-emission scanning electron microscopy (FE-SEM) revealed the formation of ribbon-shaped particles, Fig. 1 1 a. The average ribbon size was calculated statistically from FE-SEM images, measuring the length and the width of 150 particles of the same sample. The calculated average length was $3.2 \pm 1.9 \mu \mathrm{m}$ with the median of $2.8 \mu \mathrm{m}$, and the average width was $0.8 \pm 0.3 \mu \mathrm{m}$ with the median of $0.8 \mu \mathrm{m}$.

The X-Ray powder diffraction pattern of the ribbons showed packing of $\mathrm{Mn}_{12}$ molecules that was different from the initial $\mathrm{Mn}_{12}$-Ac crystals, Fig. 2. In order to grow single crystals of sufficient size to perform the single- 


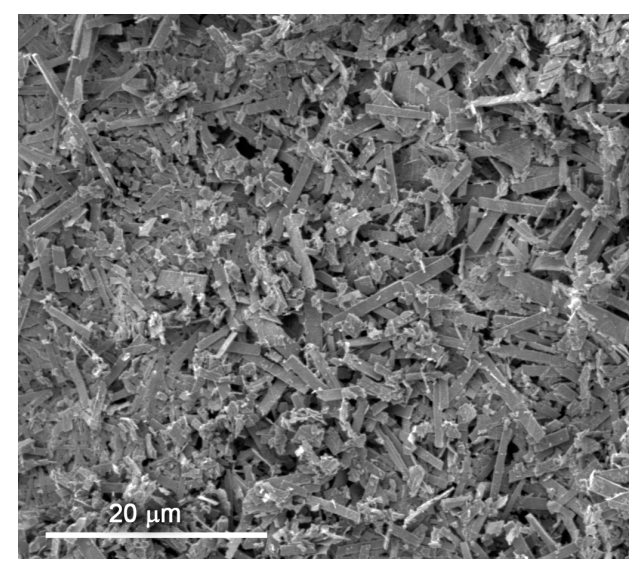

a

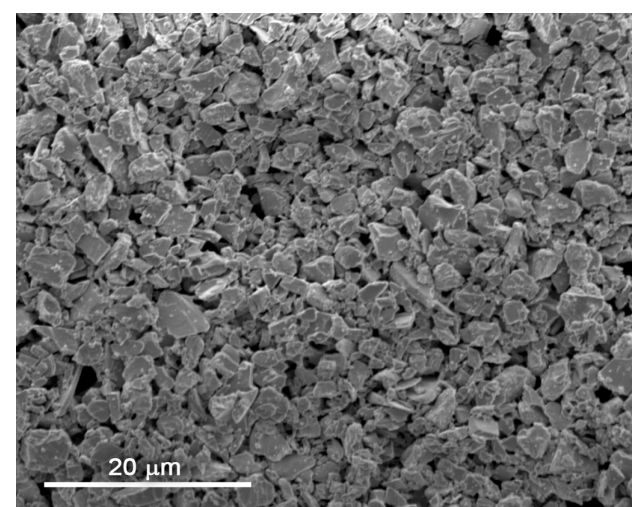

$\mathrm{b}$

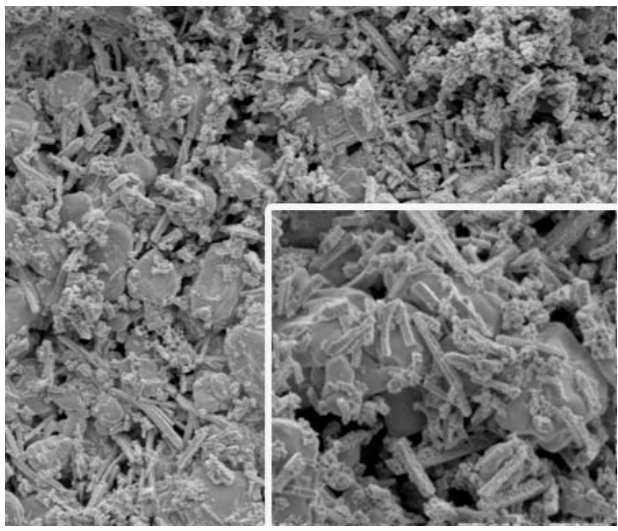

Figure 1: a) FE-SEM image of $\mathrm{Mn}_{12}$-acetate ribbons. b) FESEM image of $\mathrm{YBaCuO}$ particles. c) FE-SEM image of the mixture of $\mathrm{Mn}_{12}$-acetate ribbons and $\mathrm{YBaCuO}$ particles.

crystal X-ray diffraction experiment the synthesis procedure was further modified. The initial $\mathrm{Mn}_{12}$-acetate crystals were dissolved in acetonitrile, filtered, and subjected to slow diffusion of toluene vapors. After a few days, we observed the formation of rectangular shaped crystals. Due to their small size the single crystal X-ray diffraction experiment was performed under Synchrotron radiation in the XALOC beamline at the ALBA synchrotron. The powder pattern simulated from the resolution of the crystalline structure was in line with the powder diffractogram obtained from the ribbons, Fig 2 . The intramolecular structure was identical to that in a conventional $\mathrm{Mn}_{12}$-Ac crystal of tetragonal symmetry. However, the intermolecular packing corresponded to the triclinic space group.

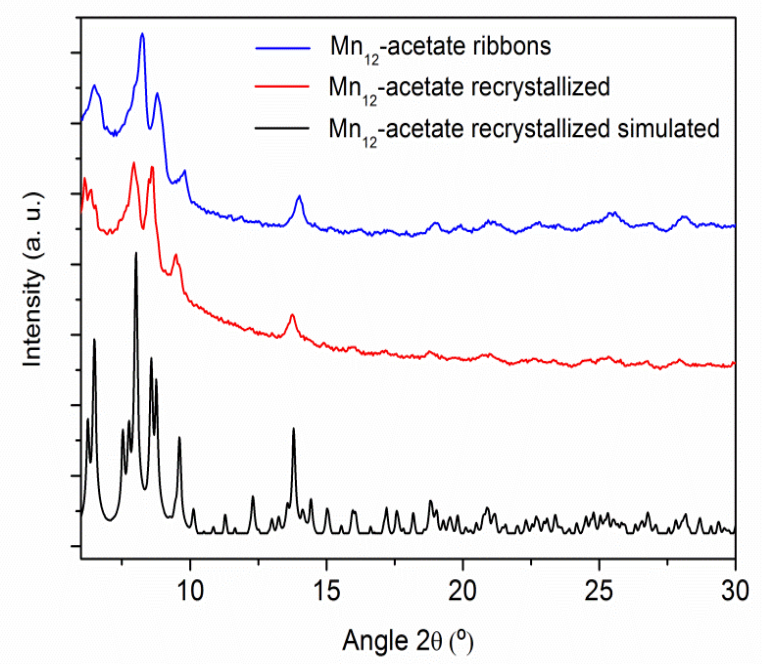

Figure 2: Color online: X-ray data from $\mathrm{M}_{12}$ ribbons (blue), from triclinic crystal grown as described in the text (red), and simulated X-ray pattern from a triclinic crystal (black).

Synthesis of the mixtures began with the preparation of a suspension of $\mathrm{Mn}_{12}$-acetate particles in $3 \mathrm{~mL}$ of toluene and a suspension of $\mathrm{YBaCuO}$ particles in the same amount of toluene. The size of $\mathrm{YBaCuO}$ particles, $2.9 \pm 1.3 \mu \mathrm{m}$, was calculated statistically from FE-SEM images, see Fig. 1 $\mathrm{b}$ b. Mixtures with different weight ratio were prepared by mixing the two suspensions under continuous stirring for 15 minutes. The resulting mixtures were filtered and the collected granular solids were ground for 10 minutes to achieve an intimate mixing of $\mathrm{Mn}_{12}$-acetate ribbons with $\mathrm{YBaCuO}$ particles. FieldEmission Scanning Electron Microscopy (FE-SEM) images demonstrated coexistence of both type of particles in the resulting mixtures, see Fig. 1 1 c.

Earlier we demonstrated ${ }^{22}$ that neither single crystals nor oriented microscrystals were needed to observe resonant spin-tunneling in molecular magnets. The tunneling maxima can be convincingly detected by plotting the field derivative of the magnetization curve measured in non-oriented or even amorphous microcrystals if the structure of the magnetic core of the $\mathrm{Mn}_{12}$ molecules remains robust with respect to the local arrangement of molecules, which is the case for the ribbons. Fig. 3 shows field derivative of magnetization curves of $\mathrm{Mn}_{12^{-}}$ acetate ribbons taken at different temperatures, and the field derivative of the magnetization curve of the sample consisting of pure $\mathrm{YBaCuO}$ grains. They clearly demonstrate that we deal with conventional $\mathrm{Mn}_{12}$ molecules and conventional $\mathrm{YBaCuO}$ superconductor.

Zero-field-cooled magnetization of curve of the 1:1 


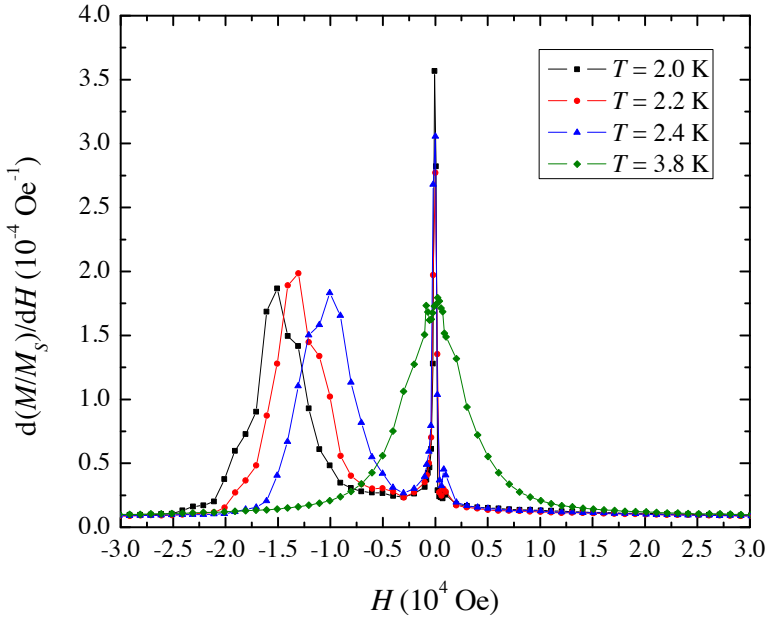

a

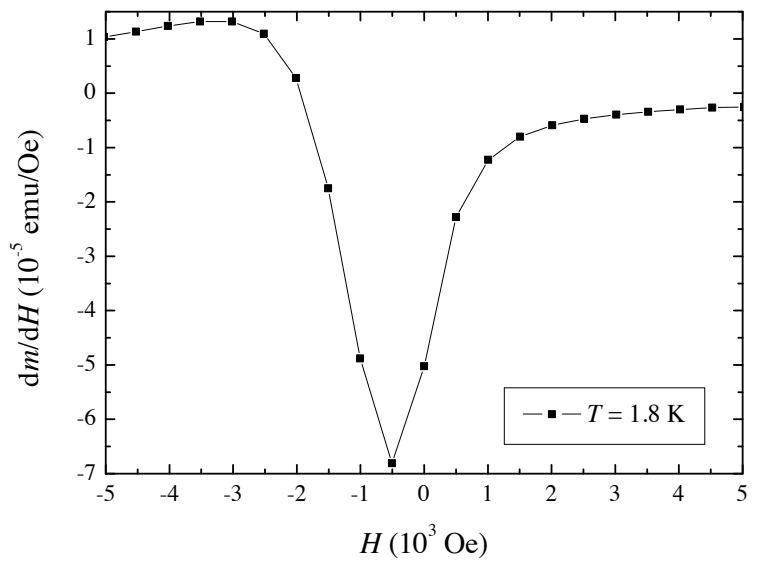

Figure 3: a) Field derivative of magnetization curves of the sample consisting of pure $\mathrm{Mn}_{12}$ ribbons at different temperatures. Presence of conventional spin-tunneling maxima separated by $0.46 \mathrm{~T}$ is apparent. b) Field derivative of the magnetization curve of the sample consisting of pure $\mathrm{YBaCuO}$ grains.

(equal mass) mixture of $\mathrm{Mn}_{12}$-acetate ribbons and $\mathrm{YBaCuO}$ particles is presented in Fig. 4-a. It shows a pronounced conventional $\mathrm{Mn}_{12}$ blocking maximum at $3.5 \mathrm{~K}$ and the absence of any second species of molecules with a different spin or a different magnetic anisotropy barrier. The paramagnetic moment of the ribbons is superimposed on the negative diamagnetic moment of $\mathrm{YBaCuO}$, which makes the total moment negative. Fig. 4-b shows a typical magnetic relaxation of the 1:1 mixture at low field. The sample was initially magnetized in a 3 - T field at $T=2 \mathrm{~K}$. The field was first taken down to $1 \mathrm{kOe}$ and then rapidly reduced to zero and switched to -20 Oe, at which time the magnetization measurements were taken. The relaxation curve indicates an unusual for $\mathrm{Mn}_{12}$ acetate, very rapid, large decrease of the magnetization on the time scale of a few minutes.

Low-field part of the descending branch of the mag-

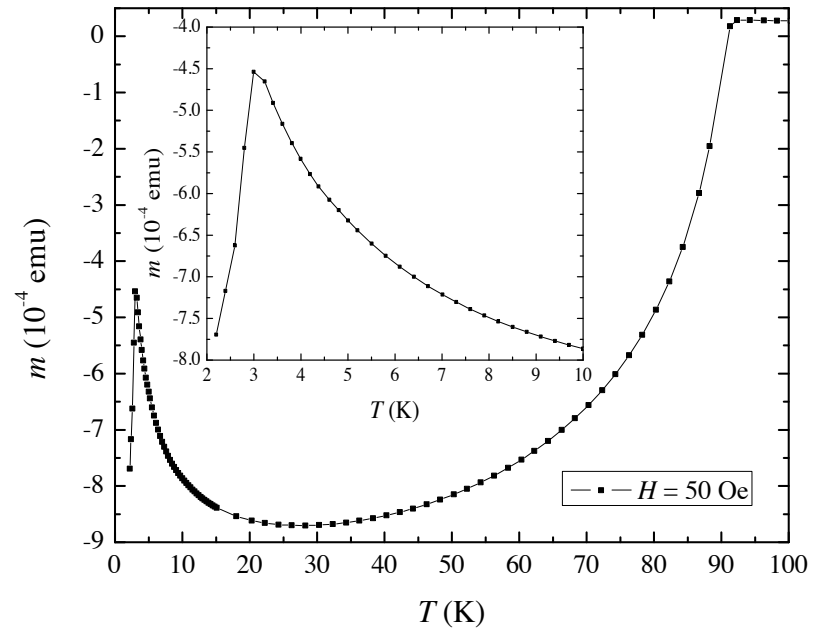

a

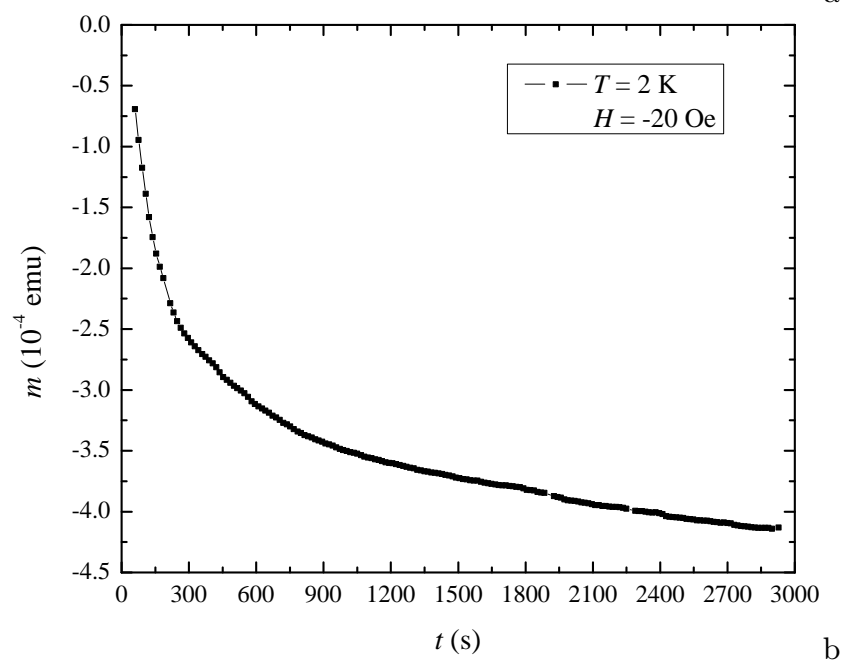

Figure 4: a) Zero-field-cooled magnetization curve of the 1:1 (equal masses) mixture of $\mathrm{Mn}_{12}$-Ac ribbons and $\mathrm{YBaCuO}$ particles at 50 Oe. The low-temperature paramagnetic peek (shown in the inset) is due to the ribbons. It is superimposed on the diamagnetic signal from the superconductor. b) Magnetic relaxation from saturation to -20 Oe at $2.0 \mathrm{~K}$.

netization curve of the $1: 1$ mixture is shown in Fig. 5-a. It represents an unusually large jump from the positive to negative magnetization in a narrow field interval from 20 Oe to -20 Oe. Such jumps have only been seen in molecular magnets under the conditions of magnetic deflagration - propagation of the front of combustion of the Zeeman energy which is similar to the combustion of a chemical substance ${ }^{819}$. However, in a zero field the Zeeman energy is zero and deflagration is not possible. Consequently, one has to assume that the jump is due to the unusually strong thermally-assisted quantum spin tunneling between matching spin levels. The derivative of the magnetization curve depicted in Fig. 5 -b shows that the width of the jump at half-height is in the ball 
park of 25 Oe, which is smaller than the width of the zero-field resonance in the conventional pure $\mathrm{Mn}_{12}$ acetate by more than one order of magnitude. The two maxima in Fig. 5tb represent field derivatives of the descending (higher maximum) and ascending (lower maximum) of the magnetization curves. The ascending maximum is lower because a fewer number of molecules hold metastable direction of their magnetic moment after going through the descending branch. Note that in the field region of the maxima the magnetization of $\mathrm{YBaCuO}$ has a negative minimum (see Fig. 3-b), which means that the maxima due to the resonant spin tunneling in the $\mathrm{Mn}_{12}$ ribbons are even more dramatic than they appear.
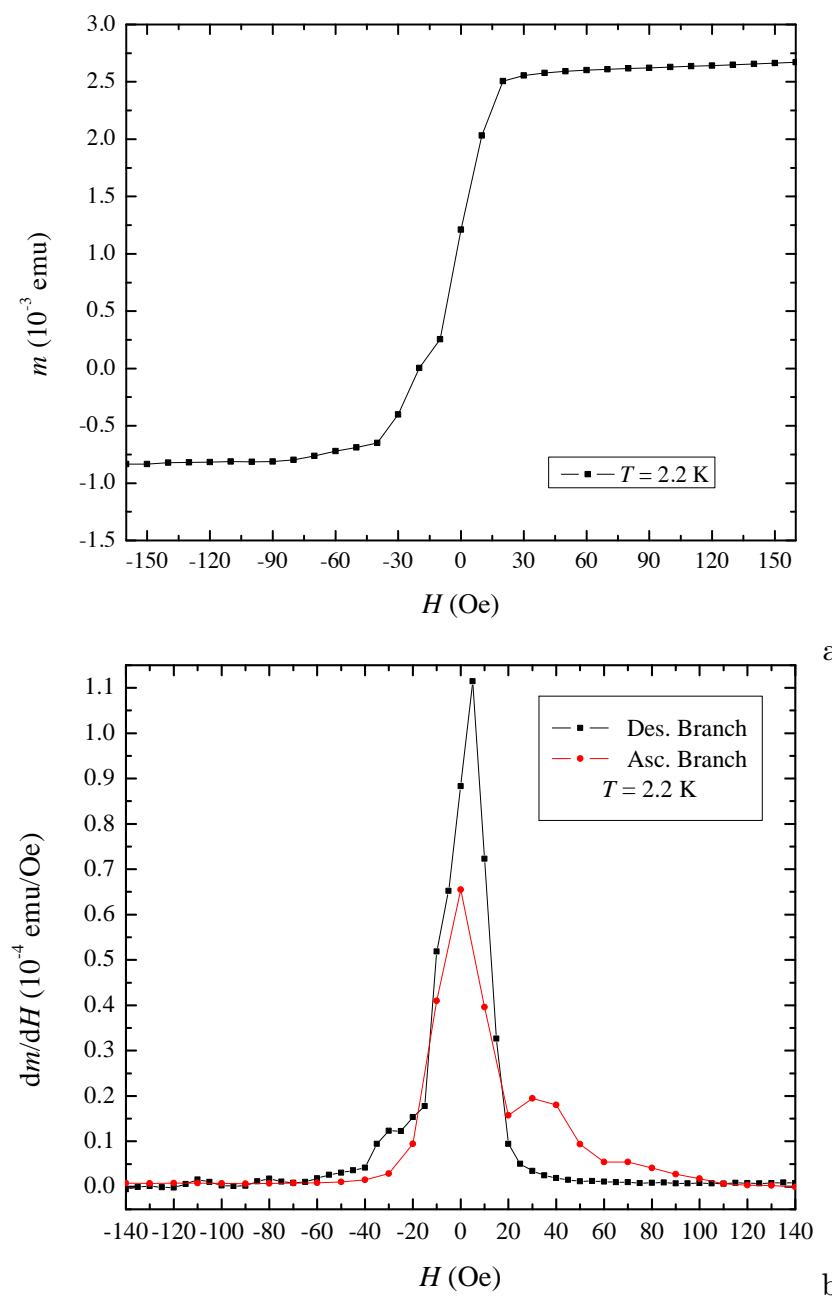

Figure 5: a) Low field part of the descending branch of the magnetization curve of the 1:1 (equal mass) mixture of $\mathrm{Mn}_{12^{-}}$ Ac ribbons and $\mathrm{YBaCuO}$ particles at $T=2.2 \mathrm{~K}$. b) Field derivative of the descending (upper maximum) and ascending (lower maximum) of the magnetization curve.

We should emphasize that the above data represent a small cut of the data accumulated by us on mixtures of different composition, taken at different temperatures, under different field-sweep protocols. The conclusion about the narrow width of the zero-field spin-tunneling resonance is consistent throughout all data. We have checked that the observed magnetic relaxation comes entirely from the $\mathrm{Mn}_{12}$ ribbons and not from the flux (if any) trapped in the superconducting grains. The sample prepared of $\mathrm{YBaCuO}$ grains alone showed no detectable relaxation at any temperature used in experiment.

The only reasonable explanation to these findings is the screening of the dipolar fields in $\mathrm{Mn}_{12}$ ribbons by superconducting currents. The irregular shape of the $\mathrm{YBaCuO}$ grains creates weak Josephson links between them in a compressed solidified mixture. At large field the links are broken by the field and the magnetization is a sum of the paramagnetic signal from the $\mathrm{Mn}_{12}$ ribbons and diamagnetic signal from decoupled superconducting $\mathrm{YBaCuO}$ grains. As the field goes down and reaches a few tens of Oe the weak links come into play. Josephson currents begin to flow between the grains, screening the dipolar fields generated by the magnetic moments of the ribbons. As a result the dipolar broadening of the zerofield spin-tunneling resonance becomes suppressed. Resonances between spin energy levels with magnetic quantum numbers $\pm m$ are restored and thermally assisted quantum tunneling becomes greatly enhanced.

In conclusion, we have demonstrated that mixing of a molecular magnet with a superconductor results in a significant narrowing of spin tunneling resonances due to the screening of dipolar fields by superconducting currents.

The work at the University of Barcelona has been supported by the Spanish Government Project No. MAT2011-23698. A.G.-S. acknowledges support from Universitat de Barcelona. I.I. and J.E. thank the MINECO for the Ramón y Cajal contract and the FPI fellowship, respectively. The work of EMC at CUNY Lehman College has been supported by the Office of Science of the U.S. Department of Energy through grant No. DE-FG02-93ER45487.
1 See recent book: Molecular Magnets - Physics and Applications, Ed. by J. Bartolomé, F. Luis., and J. F. Fernández, (Springer, 2014, Heidelberg New York Dordrecht London; DOI: $10.1007 / 978-3-642-40609-62014)$.

2 J. Tejada, E. M. Chudnovsky, E. del Barco, J. M. Hernandez, and T. P. Spiller, Nanotech. 12, 181 (2001).

3 J.R. Friedman, M.P. Sarachik, J. Tejada, and R. Ziolo,
Phys. Rev. Lett. 76, 3830 (1996).

4 J.M. Hernandez, X.X. Zhang, F. Luis, J. Bartolome, J. Tejada, and R. Ziolo, Europhys. Lett. 35, 301 (1996).

${ }^{5}$ L. Thomas, Fl. Lionti, R. Ballou, D. Gatteschi, R. Sessoli, and B. Barbara, Nature London 383, 145 (1996).

${ }^{6}$ E.M. Chudnovsky and J. Tejada, Macroscopic Quantum Tunneling of the Magnetic Moment, (Cambridge Univer- 
sity Press, Cambridge, England, 1998).

7 W. Wernsdorfer and R. Sessoli, Science 284, 133 (1999).

${ }^{8}$ Y. Suzuki, M.P. Sarachik, E.M. Chudnovsky, S. McHugh, R. Gonzalez-Rubio, N. Avraham, Y. Myasoedov, E. Zeldov, H. Shtrikman, N.E. Chakov, and G. Christou, Phys. Rev. Lett. 95, 147201 (2005).

9 A. Hernandez-Minguez, J.M. Hernandez, F. Macia, A. Garcia-Santiago, J. Tejada, and P.V. Santos, Phys. Rev. Lett. 95, 217205 (2005).

10 C. Schlegel, J. van Slageren, M. Manoli, E. K. Brechin, and M. Dressel, Phys. Rev. Lett. 101, 147203 (2008).

11 S. Bertaina, S. Gambarelli, T. Mitra, B. Tsukerblat, A. Müller, and B. Barbara, Nature (London) 453, 203 (2008); ibid 466, 1006 (2010).

12 M. Ganzhorn, S. Klyatskaya, M. Ruben, and W. Wernsdorfer, Nat. Nano. 8, 165 (2013).

13 M. Ganzhorn, S. Klyatskaya, M. Ruben, and W. Wernsdorfer. ACS Nano. 7, 6225 (2013).

14 E. M. Chudnovsky and D. A. Garanin, Phys. Rev. Lett. 89, 157201 (2002).
15 J. Tejada, R. Amigo, J. M. Hernandez, and E. M. Chudnovsky, Phys. Rev. B 68, 014431 (2003).

16 M. Bal, J. R. Friedman, K. Mertes, W. Chen, E. M. Rumberger, D. N. Hendrickson, N. Avraham, Y. Myasoedov, H. Shtrikman, and E. Zeldov, Phys. Rev. B 70, 140403(R) (2004)

17 K. Park, M. A. Novotny, N. S. Dalal, S. Hill, and P. A. Rikvold, Phys. Rev. B 65, 014426 (2001).

18 J. R. Friedman, M. P. Sarachik, R. Ziolo, Phys. Rev. B 58, 14729(R) (1998).

19 E. M. Chudnovsky and J. R. Friedman, Phys. Rev. Lett. 85, 5206 (2000).

20 I. Imaz, F. Luis, C. Carbonera, D. Ruiz-Molina, and D. Maspoch, Chem. Commun. 2008, 1202 (2008).

21 T. Lis, Acta Crystallogr. B 36, 2042 (1980).

22 S. Lendínez, R. Zarzuela, J. Tejada, M. W. Terban, S. J. L. Billinge, J. Espin, I. Imaz, D. Maspoch, and E. M. Chudnovsky, Phys. Rev. B 91, 024404 (2015). 